\title{
Molecular Mechanisms of Blood Clotting
}

Although the complex sequences of biochemical reactions involved in blood clotting are well known, there has been less agreement about proposals for a mechanism in terms of the three-dimensional interactions between molecules of known structure; much is known about what happens without understanding how. Progress towards bridging this gap between a biochemical and a molecular biological description of blood clotting is represented in the report by Tooney and Cohen on page 23 of this issue of Nature. By analysis of a modified fibrinogen which forms microcrystals, these authors conclude that the fibrinogen-fibrin conversion resembles a transition between two different polymorphic aggregates of monomers which incur no intra-molecular rearrangement.

Blood clotting is brought about when thrombin converts the soluble plasma protein fibrinogen into fibrin, which becomes insoluble by rapidly polymerizing into long fibres. The fibrinogen molecule consists of three pairs of identical chains of molecular weight of about 50,000 , and the conversion to fibrin involves the cleavage of the small peptides from fibrinogen. Considerable uncertainty exists about the structure of the fibrinogen molecule. It was classified by Astbury with $\alpha$-keratin, epidermin and myosin because it gave an X-ray diffraction pattern which indicated a molecular structure similar to these $\alpha$-proteins. This $\alpha$-protein structure is now known to take the form of two $\alpha$-helices coiled about each other to form a two strand rope, but, unlike the simple rod shaped muscle proteins paramyosin and tropomyosin which are 100 per cent helical, fibrinogen is much less helical and thus a large fraction of the molecule is of unknown structure.

On the basis of various techniques conflicting proposals have been made that fibrinogen is a long thin molecule, that it consists of three globular portions linked by two helical rods, that it has an open spherical lattice structure, that the fibrino-peptides are close together or alternatively far apart. In electron micrographs obtained by shadow casting the fibrinogen molecule appears to be about $475 \AA$ long, whereas preparations of fibrin have an axial periodicity of $225 \AA$; the conflicting explanations involve shrinkage of the molecules or staggering the molecules by half their length axially. Although Tooney and Cohen's report does not solve all of these problems, considerable light is thrown on the likely molecular mechanisms involved in clotting.

Tooney and Cohen have discovered that when fibrinogen is treated with certain bacterial proteases, part of the molecule is cleaved to yield fibrinogen Ps which is still convertible to fibrin by thrombin; the heaviest of the three chains comprising the molecule is affected. At high ionic strength fibrinogen, fibrinogen Ps and fibrin can all yield similar structures with axial periodicities of $225 \AA$ A. At lower ionic strength fibrinogen forms fibres with no visible periodicity in the electron microscope and fibrinogen Ps precipitates as highly ordered rod-shaped patterns, termed as obliquely striated and orthogonal sheet, both with transverse bands separated axially by
$450 \AA$. Because alternate bands are shifted laterally with respect to each other a true axial repeat of $900 \AA$ is generated in both cases. The authors infer head to tail aggregation of molecules $450 \AA$ long. Furthermore, in view of the similarity between the high ionic strength structures given by fibrin Ps (that is, fibrinogen Ps precipitated with thrombin) and native fibrin they infer close similarity between the molecular structures of fibrinogen and fibrinogen Ps.

Perhaps the most interesting observation of Tooney and Cohen, however, is that superposition of variously translated images of the orthogonal sheet structure can produce a number of patterns similar to some intermediate patterns observed and, in particular, to the native fibrin pattern with a period of $225 \AA$. This suggests that there may be one fundamental array, say the orthogonal sheet, which by aggregating in specific modes can generate various structures. Thus fibrin, say Tooney and Cohen, may be a polymorphic form of fibrinogen, and the transition between the two would be accompanied by a minimal intra-molecular rearrangement.

The fact that a modified fibrinogen can adopt a regular microcrystalline form holds out promise that further details of the molecular structure and its possible interactions will be determined so that theories on the molecular mechanism of blood clotting can be tested.From a Correspondent.

\section{I and B Cell Cooperation}

IMMUNOBIOLOGY is in the middle of a storm and the confusion is considerable. Three articles in the current issue of Nature New Biology (May 3) all exemplify what is happening; they all concern in one way or another the cooperation between $\mathbf{T}$ and $\mathbf{B}$ cells, a theme which is currently of great interest is cellular immunology.

Schimpl and Wecker (Nature New Biology, 237, 15 ; 1972) studied the response of mouse spleen cells to stimulation by sheep red blood cells (SRBC) in vitro. In their first experiments they established that $T$ cells were a prerequisite for an immune response measurable as $19 \mathrm{~S}$ plaque forming cells. They did this by showing that spleen cells treated before culture with anti- $\theta$ antisera were unresponsive, but they found that the supernatant from simple spleen cultures could occasionally make an inadequately anti- $\theta$ treated cell population respond quite well to SRBC. These supernatants were always inactive on spleen cells that had been rigorously treated with anti-theta. Schimpl and Wecker therefore conclude that spleen cell populations in vitro can liberate a $\mathrm{T}$-cell expanding factor (TEF) which is perhaps similar in its effect to the twin stranded RNA which Cone and Johnson (J. Exp. Med., 133, $665 ; 1971$ ) have shown can expand the influence of thymocytes in vivo.

Schimpl and Wecker went on to experiment with supernatants from mixed cultures in which spleens from two different strains of mice had been growing for twenty- 